\title{
Dynamic Reconfiguration of Functional Topology in Human Brain Networks: From Resting to Task States
}

\author{
Wenhai Zhang $\mathbb{D}^{1,2}$ Fanggui Tang, ${ }^{1}$ Xiaolin $\mathrm{Zhou}^{3}$ and Hong $\mathrm{Li}^{4}$ \\ ${ }^{1}$ College of Education Science, Hengyang Normal University, Hengyang 421002, China \\ ${ }^{2}$ Mental Health Center, Yancheng Institute of Technology, Yancheng 224051, China \\ ${ }^{3}$ School of Psychology and Cognition, Peking University, Beijing 100871, China \\ ${ }^{4}$ Institute for Brain and Psychological Sciences, Sichuan Normal University, Chengdu 610066, China \\ Correspondence should be addressed to Wenhai Zhang; zwh2007106@126.com
}

Received 4 May 2020; Revised 23 July 2020; Accepted 26 August 2020; Published 8 September 2020

Academic Editor: Fushun Wang

Copyright $@ 2020$ Wenhai Zhang et al. This is an open access article distributed under the Creative Commons Attribution License, which permits unrestricted use, distribution, and reproduction in any medium, provided the original work is properly cited.

\begin{abstract}
Task demands evoke an intrinsic functional network and flexibly engage multiple distributed networks. However, it is unclear how functional topologies dynamically reconfigure during task performance. Here, we selected the resting- and task-state (emotion and working-memory) functional connectivity data of 81 health subjects from the high-quality HCP data. We used the network-based statistic (NBS) toolbox and the Brain Connectivity Toolbox (BCT) to compute the topological features of functional networks for the resting and task states. Graph-theoretic analysis indicated that under high threshold, a small number of long-distance connections dominated functional networks of emotion and working memory that exhibit distinct long connectivity patterns. Correspondently, task-relevant functional nodes shifted their roles from within-module to between-module: the number of connector hubs (mainly in emotional networks) and kinless hubs (mainly in working-memory networks) increased while provincial hubs disappeared. Moreover, the global properties of assortativity, global efficiency, and transitivity decreased, suggesting that task demands break the intrinsic balance between local and global couplings among brain regions and cause functional networks which tend to be more separated than the resting state. These results characterize dynamic reconfiguration of large-scale distributed networks from resting state to task state and provide evidence for the understanding of the organization principle behind the functional architecture of task-state networks.
\end{abstract}

\section{Introduction}

Understanding how the brain shapes mind, such as cognition and emotion, ultimately relies on the knowledge of largescale brain networks [1]. The Human Connectome Project (HCP) used high-quality neuroimaging to map the structural and functional connectivity of the normal human brain [2], which provides new opportunity to understand general topological principles of brain network organization. Graph theory-based connectivity research has shown that a brain network is composed of functionally separate subnetworks or modules [3]. As a complex system, the brain flexibly processes multiple incoming information through interplaying between distributed subsystems $[4,5]$. Moreover, the intrinsic functional network during resting state primarily shapes a standard architecture of task-based functional brain organi- zation and is secondarily evoked by task-relevant networks [6]. However, little is known about how the functional topology dynamically reconfigures for task performance.

In graph-theoretic research, a function network is described as a graph with a collection of nodes representing brain regions and edges representing functional interactions in the brain $[7,8]$. Nodes are further grouped into module or community with highly connected within-group links and a minimally possible number of between-group links [9]. Human brain networks have complex local and global topological properties (e.g., hub nodes, modules, transitivity measuring functional separation, and global efficiency measuring functional integration) [8]. When task demands change from resting state to task state, metabolic energy is necessarily redistributed to support the reorganized functional architecture [10] and the functional network is 
dynamically reorganized according to the specific cognitive demands of the task [11]. Correspondently, functional topologies such as connections between/within modules, nodal features, and global features (e.g., transitivity and global efficiency) are reconfigured [12-14]. However, there are still the following three unsolved questions.

First, there is lack of enough evidences to determine how long connections switch from resting state to task states, e.g., emotion and working memory (WM). The functional connectivity refers to some forms of statistical dependency between nodes, and short-distance links are distributed mainly within communities while long-distance links are distributed between communities [15, 16]. During tasks, short connections within communities decrease while long connections between communities increase [6, 17]. A recent meta-analysis indicated that the coactivation networks elicited by a wide range of tasks have more long-range connections [18]. Particularly, the default mode network (DMN) actively contributes to function integration [19]: intraDMN connectivity decreased while inter-DMN connectivity increased during a 2-back versus a 1-back working memory (WM) task [13]. Moreover, emotion processing (e.g., reappraisal) produces distributed alterations in functional connections involving visual, dorsal attention, frontoparietal, and DMN modules [20]. Long connections between communities are particularly important for brain function because they are responsible for intermodular communication $[12,21]$. However, performing statistical testing on connectivity values for large networks suffers from multiple comparison problem so that long-links are easily ignored because of their weak connectivity values [7, 22]. The network-based statistic (NBS) has greater power to detect a whole cluster of regions spanning multiple connections and makes it possible to find a set of connections forming a subnetwork associated with an experimental effect [23]. Here, we used the NBS to further clarify how long connections change during the WM and emotional task versus the resting state.

Second, it is unclear how functional hubs dynamically change their nodal roles during the WM and emotional tasks. Resting-state fMRI research has demonstrated functional hubs distributing in the heteromodal association cortex (e.g., the precuneus, posterior and anterior cingulate gyrus, ventromedial frontal cortex, and inferior parietal regions) $[3,24]$. Hubs flexibly process multiple information and rapidly update their connectivity pattern according to task demands $[25,26]$. Hub nodes are generally divided into three different roles: provincial hubs with the vast majority of links within their module, connector hubs with many links to most of the other modules, and kinless hubs with links homogeneously distributed among all modules [27]. Finc et al. [13] found that the number of connector hubs increased whereas the number of provincial hubs decreased when the WM task became more demanding. Moreover, task-relevant nodes within auditory, visual, salience, and context community become activated in the WM task while subcortical regions (e.g., amygdala and putamen) take an important role in emotional tasks [20, 26, 28-30]. However, the previous studies ignored the shifting of nonhubs to hubs and rarely mentioned kinless hubs.
Third, it is unclear whether intrinsic functional networks become more integrated or separated during the shift from resting state to task state. A number of structural and resting-state fMRI studies have indicated that brain networks exhibit economical small-world topology [31-33], balancing integration and segregation between brain regions [34, 35]. To satisfy ever changing task demands, the global properties (e.g., clustering and modularity) of brain network organization are responsive to the changing task contexts [12]. Some studies have found that functional networks tend to be of higher global network integration at task state: for example, the performance of cognitive tasks (including WM) is associated with increased global efficiency and less segregation of processing relative to resting state $[36,37]$. Other studies have proposed that the global topological properties are largely invariant in order to continually maintaining the balance of efficient local and global processing [38, 39]. Another studies demonstrated that functional networks tend to be highly separated (e.g., negative assortativity coefficients) and exhibit a more random configuration at higher levels of task difficulty (e.g., emotional task) [8, 30, 40]. This inconsistency might be because of multiple factors such as different tasks, different signal natures of fMRI and EEG, or different ways to constructing function networks. More high-quality researches are pressed to clarify the consistency.

To address the three questions, we selected the restingstate denoised by FIX (FMRIB's ICA-based X-noiseifier) and task-state (EMOTION and WM) fMRI data from the HCP data with 500 subjects (see Methods for details). Then, we used Pearson's correlation to separately construct three functional networks (FIX, EMOTION, and WM) for each subject. Next, we performed connectivity analysis for EMOTION and WM versus FIX using the NBS toolbox [23] to determine how long connections change during task states versus resting state. We also used the Brain Connectivity Toolbox (BCT) to compute nodal features of participant index (PI) and within-module $Z$-score and global properties (assortativity, global efficiency, and transitivity) and then performed one-way ANOVA with 3 conditions (FIX, EMOTION, and WM) for global and nodal properties at each threshold of $5-15 \%$. Considering that functional networks at task state need to exchange multiple information between different communities, we firstly predicted that although long connections are of a small proportion, they would become more significant relative to resting state because they are responsible for intermodular communication [12, 21]. We also predicted that with the increase of task demands, the number of task-relevant connector and kinless hubs would increase while the number of provincial hubs would decrease [13]. Finally, we predicted that under the disturbance of active tasks, the balance between integration and segregation at resting state would be disrupted and functional networks would tend to be more separated and randomized $[8,30,40]$.

\section{Methods}

2.1. Participants. After registering an account at ConnectomeDB and agreeing to the Open and Restricted Access Data 
Use Terms (http://www.humanconnectome.org/), we were approved to download the HCP data with 500 subjects. After matching age with gender and excluding twins, we selected 81 right-handed healthy adults (age 22-30 years old; 40 males and 41 females). These participants had no prior history of neurological or psychiatric disorders.

2.2. fMRI Data Selection and Processing. The detailed data acquisition and experimental procedure were described at the HCP website [2]. For structural imaging, T1w was acquired using a 32-channel head coil and 3T Siemens product (MPRAGE and SPACE) sequences $(\mathrm{TR}=2400 \mathrm{~ms}$, $\mathrm{TE}=2.14 \mathrm{~ms}$, flip angle $=8$ degrees, $\mathrm{FOV}=224 \times 224 \mathrm{~mm}$, voxel size $=0.7 \mathrm{~mm}$ isotropic). The selected HCP data included the resting-state and task-state fMRI image datasets. The resting-state fMRI data were acquired in four runs of approximately 15 minutes each, two runs in one session and two in another session, with eyes open with relaxed fixation on a projected bright cross hair on a dark background (and presented in a darkened room) $(\mathrm{TR}=720 \mathrm{~ms}, \mathrm{TE}=$ $33.1 \mathrm{~ms}$, flip angle $=52$ degrees, $\mathrm{FOV}=208 \times 108$, matrix $=$ $104 \times 90$, slice thickness $=2 \mathrm{~mm}$ isotropic). Following completion of resting-state fMRI in each of the two fMRI scanning sessions, the task-state data were acquired with the same EPI pulse sequence parameters as the resting-state fMRI. These subject-specific images had been preprocessed through the HCP Minimal Processing Pipelines (MPP): (1) to remove spatial artifacts and distortions; (2) to generate cortical surfaces, segmentations, and myelin maps; (3) to make the data easily viewable in the Connectome Workbench visualization software; (4) to generate precise withinsubject cross-modal registrations; (5) to handle surface and volume cross-subject registrations to standard volume and surface spaces; and (6) to make the data available in the CIFTI format in a standard grayordinate space (see [41] for details).

The task-state fMRI data included an emotion processing task and a WM task. The emotion processing task is a Hariri matching task [42], in which the participants were asked to decide which of the two faces presented at the bottom of the screen matched the face at the top of the screen or which of two shapes presented at the bottom of the screen matched the shape at the top of the screen [43]. The faces have either angry or fearful expressions and simple geometric shapes (circles, vertical, and horizontal ellipses) were used as control stimuli. The WM task is an $n$-back task in which 4 different stimulus types (face, places, tools, and body parts) are presented in separate blocks within each run. Within each run, $1 / 2$ of the blocks use a 2-back WM task and 1/2 of the blocks use a 0 -back WM task. Each of the two runs contains 8 task blocks (10 trials of $2.5 \mathrm{~s}$ each, for $25 \mathrm{~s}$ ) and 4 fixation blocks (15 s each).

Following Cao et al. [44], the mean average of all taskrelated signal fluctuations was removed by regression with separate regressors for each experimental condition in order to only account for condition-specific effects, prior to graph construction. The parcellation with 333 parcels developed by Gordon et al. [45] was combined with subcortical areas (bilateral amygdala, hippocampus, accumbens, caudate, pal- lidum, putamen, thalamus, ventral diencephalon, cerebellum, and the whole brain stem) into a new parcellation with the 352 functional parcels (downloading from https:// sites.wustl.edu/petersenschlaggarlab/resources/). Then, we used Connectome Workbench developed by the HCP (http://www.humanconnectome.org/software/connectomeworkbench.html) to extract the 352 parcels' time series from the residual task-fMRI data and merged the time series of two scanning orders. Next, we computed the pairwise Pearson's correlation matrices of all these parcel time series for each task. Finally, we removed the rows and columns corresponding to 47 parcels with no original labels in the parcellation developed by Gordon et al. [45] and thus obtained the functional networks with the size $305 \times 305$.

The resting-state fMRI data contained the FIX data. During the preprocessing, the FIX data had been cleaned of structured noise by a new approach that combines ICA with a more complex automated component classifier referred to as FIX [41]. Similar to the task state, we obtained Pearson's correlation matrices with 305 functional nodes for the FIX data.

2.3. Network Connection Analysis. To identify network connections that varied with the task demand, we used the NBS approach [23]. Full-linking connectivity matrices were entered as repeated measure-dependent variables into the NBS toolbox (freely downloaded from http://www.nitrc .org/projects/nbs/), with the contrast of EMOTION or WM versus FIX. According to Figure 1, the inflection points separately occur at the threshold of $t=4.6$ for EMOTION versus FIX (i.e., the number of connected edges decreases more sharply when $t<4.6$; the curve nearly parallels with $t$-axis when $t>4.6)$ and at the threshold of $t=6.4$ for WM versus FIX (i.e., the curve nearly parallels with $t$-axis when $t>6.4$ ). Moreover, the networks for EMOTION and WM versus FIX hold comparable edges at these inflection points. Therefore, an individual-connection-level threshold of $t=4.6$ and 6.4, respectively, for EMOTION versus FIX and WM versus FIX was used with extent-based correction for multiple comparisons, 5000 permutations, and an overall corrected $p<0.0001$.

2.4. Graph-Theoretic Processing. After these correlation networks were Fisher- $Z$ transformed, their diagonal elements and negative connections were set to zero. We used the BCT (http://www.brain-connectivity-toolbox.net) to sparse functional networks in 1\% interval from the threshold 5\% to $15 \%$. For each threshold, we constructed weighted networks for the FIX, EMOTION, and WM condition. In these weighted networks, inter/intramodal connections below the threshold were assigned to 0 while the connections above the threshold remained unchanged because weak and nonsignificant links may represent spurious connections that tend to obscure the topology of strong and significant connections and as a result are often discarded [8].

The graph analyses included nodal and global topological features for each threshold. First, to explore how the hubs change in the different task conditions, we computed the nodal PI (or participation coefficients) and within-module 


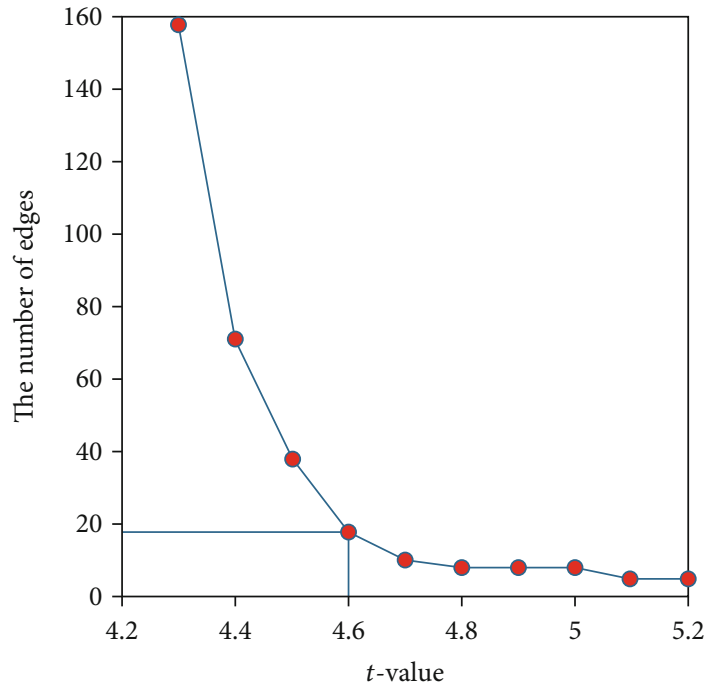

(a)

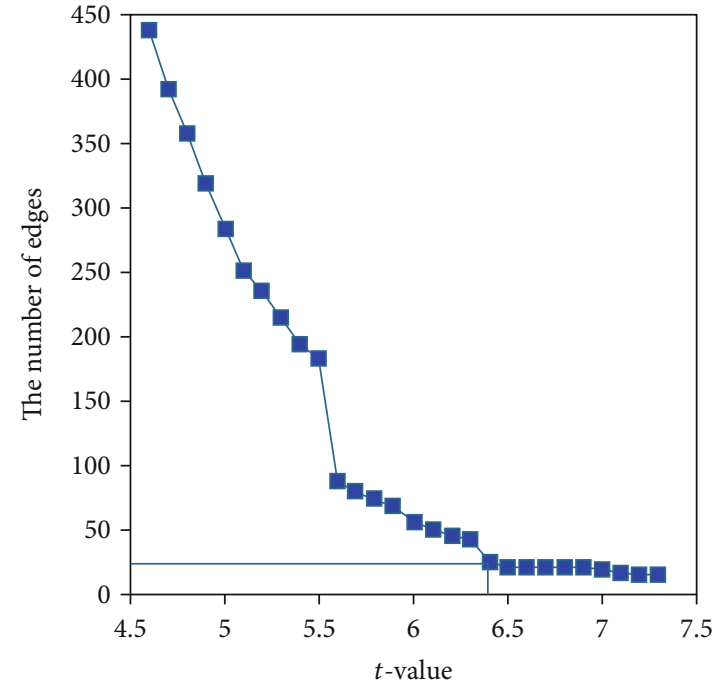

(b)

FIGURE 1: The number of connected edges varies with the threshold of the $t$-value from the network-based statistic method. (a) The inflection point occurs at the threshold of $t=4.6$ for EMOTION versus resting state. (b) The inflection point occurs at the threshold of $t=6.4$ for working memory versus resting state.
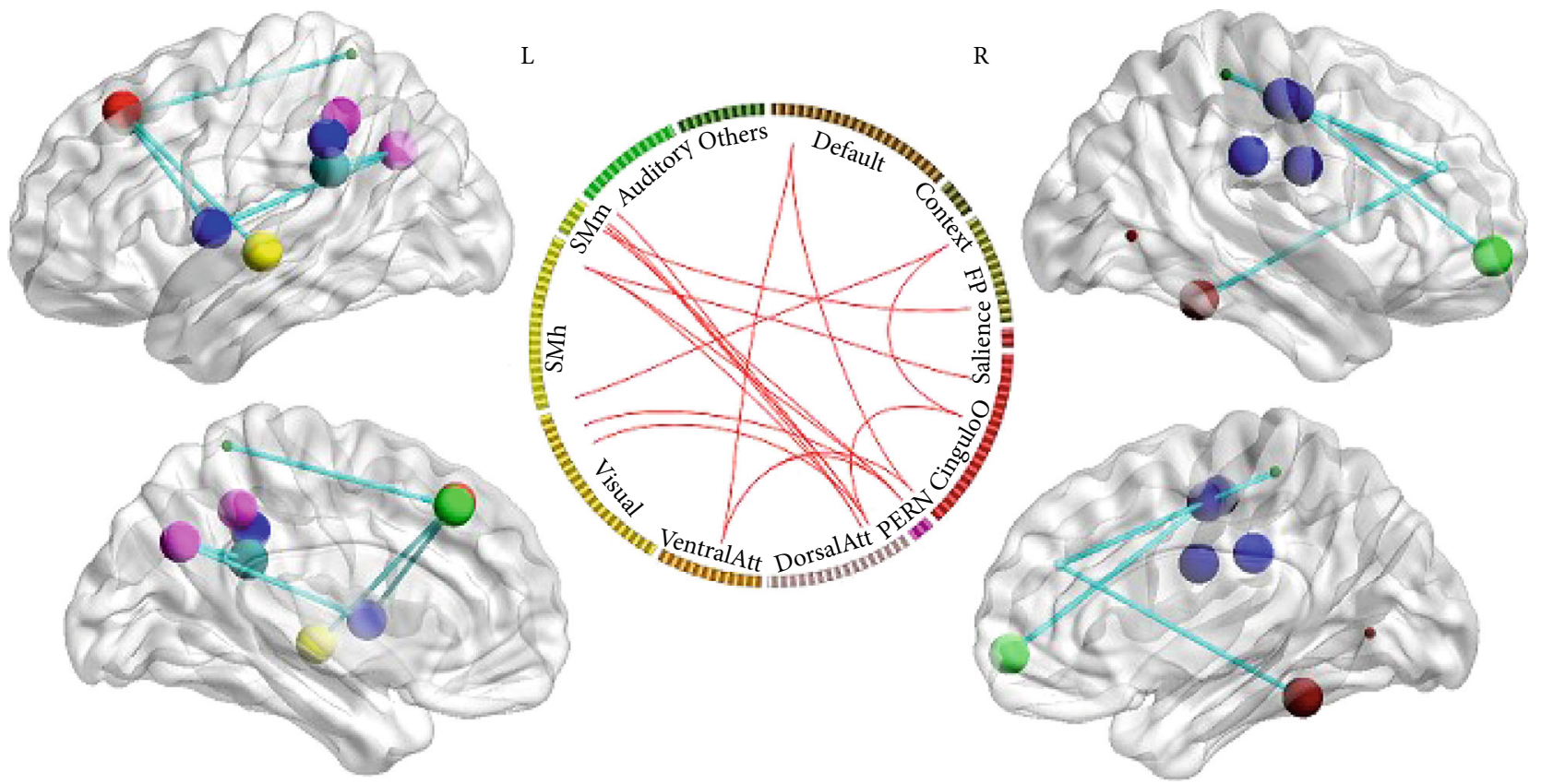

FIGURE 2: Two-dimension and three-dimension depictions of connectivity analysis result in the EMOTION versus FIX condition. The nodal color denotes affiliative community; the nodal size represents the magnitude of nodal betweenness centrality; the edge depicts binarized edge.

degree $Z$-score for each threshold. PI measures the diversity of intermodular connections of individual nodes while within-module degree $Z$-score measures the extent to which a node is connected to other nodes within its module [46]. Following Guimera et al. [27] and Finc et al. [13], we first classified nodes as hubs $\left(Z_{i}>1\right)$ and nonhubs $\left(Z_{i}<1\right)$. Then, the hubs were further divided into three classes: (1) provincial hubs with $Z_{i}>1$ and $\mathrm{PI}_{i}<0.3$; (2) connector hubs with $Z_{i}>1$ and $0.3<\mathrm{PI}_{i}<0.75$; and (3) kinless hubs with $Z_{i}>1$ and $\mathrm{PI}_{i}>0.75$.
Second, we analyzed the following global network properties to determine functional networks that become integrated or separated from resting to task states. (1) Assortativity is a correlation coefficient between the degrees of all nodes on two opposite ends of a link. A positive assortativity coefficient indicates that nodes tend to link to other nodes with the same or similar degrees. (2) Global efficiency is the inverse of the average shortest path length. (3) Transitivity is the ratio of triangles to triplets in the network and is an alternative to the clustering coefficient. 
TABLE 1: The nodes of connected network in the emotion and working-memory versus the resting-state condition during the network-based statistical analysis.

\begin{tabular}{lcccccc}
\hline ID & Parcel label & AAL & Centroid MNI & $y$ & Role* \\
\hline 6 & Default_6 & Frontal_Sup_L & -19.5 & 30.1 & 45.5 & P \\
40 & Default_40 & Frontal_Mid_R & 30.6 & 18.9 & 48.7 & P \\
41 & Default_41 & Temporal_Sup_R & 54.4 & 1.1 & -12.9 & Nonhub \\
43 & Context_2 & Calcarine_L & -8.8 & -49.8 & 4.2 & Nonhub \\
48 & Context_7 & Fusiform_R & 34.6 & -23.9 & -20.4 & Nonhub \\
52 & FrontoParietal_3 & Frontal_Sup_Media & -5.5 & 29.3 & 44 & Nonhub \\
69 & FrontoParietal_20 & Frontal_Mid_Orb_R & 28.4 & 57 & -5.1 & Nonhub \\
84 & CinguloOperc7 & SupraMarginal_L & -57.7 & -40.6 & 35.8 & P/C \\
90 & CinguloOperc_13 & Insula_L & -28.8 & 23.7 & 8.4 & P/C \\
91 & CinguloOperc_14 & Rolandic_Oper_L & -59.8 & -4.1 & 8.8 & P/C \\
94 & CinguloOperc_17 & Rolandic_Oper_L & -51.8 & -0.6 & 5 & P/C \\
117 & CinguloOperc_40 & SupraMarginal_R & 54.9 & -27 & 29.6 & P/C \\
119 & PERN_2 & Precuneus_L & -12.7 & -64.9 & 31.8 & C \\
128 & DorsalAttn_6 & Parietal_Inf_L & -42.9 & -45 & 43 & P/C \\
130 & DorsalAttn_8 & Frontal_Inf_Tri_L & -43.6 & 36.3 & 8.5 & P \\
165 & VentralAttn_11 & Temporal_Mid_L & -59 & -18 & -3 & P \\
205 & Visual_28 & Occipital_Mid_R & 31.7 & -85.7 & 2.4 & Nonhub \\
206 & Visual_29 & Lingual_R & 43.8 & -67.2 & 2 & C \\
208 & Visual_31 & Temporal_Mid_R & 49 & -54.5 & 8.8 & Nonhub \\
209 & Visual_32 & Lingual_R & 31.2 & -45.6 & -5.8 & Nonhub \\
211 & Visual_34 & Fusiform_R & 34.9 & -44 & -20 & Nonhub \\
217 & SMhand_1 & Cuneus_R & -18.8 & -48.7 & 65 & P/C \\
251 & SMhand_35 & Postcentral_R & 39.2 & -34.6 & 57.5 & P \\
260 & SMmouth_6 & Precentral_R & 42.3 & -11 & 47.3 & Nonhub \\
261 & SMmouth_7 & Postcentral_R & 53.9 & -8.3 & 26.1 & P/C \\
262 & SMmouth_8 & Precentral_R & 47.8 & -15.1 & 49.3 & P/C \\
263 & Auditory_1 & Heschl_L & -32 & -29.3 & 15.6 & Nonhub \\
264 & Auditory_2 & SupraMarginal_L & -46.3 & -41.4 & 25.9 & Nonhub \\
\hline & & & & & \\
& & & & & & \\
\end{tabular}

Note. P: provincial hub; C: connector hub. ${ }^{*}$ Nodal role at the resting state.

We wrote the custom Matlab scripts to perform one-way ANOVA with 3 conditions (FIX, EMOTION, and WM) for global and nodal properties separately in weighted networks at each threshold. The Bonferroni method was used for all post hoc analyses. Significant effects of $p<0.001$ were reported.

\section{Results}

\subsection{Connectivity-Based Analysis}

3.1.1. EMOTION versus FIX Contrast. The NBS analysis revealed that a single connected network with 18 nodes and 18 edges was altered $(t=4.60, p<0.0001$, corrected $)$ (Figure 2). Table 1 shows the nodes of the connected network. The involved nodal regions included Default_6, two FrontoParietal nodes ( 3 and 20), three CinguloOperc nodes (7, 17, and 40), PERN_2, two DorsalAttn nodes (6 and 8), one VentralAttn node (11), two Visual nodes $(11,29$, and $34)$, two SMHand nodes (1 and 35), three SMmouth nodes $(6,7$, and 8$)$, and one Auditory node (2). All of the connec- tions exhibited increased values in the EMOTION condition compared with the FIX condition. However, there were no significant connections between Context/Salience/Subcortical nodes and other nodes.

3.1.2. WM versus FIX Contrast. The NBS analysis showed a significant increase of connectivity in the WM condition compared with the FIX condition in a single brain network formed by 20 nodes and 23 edges $(t=6.40, p<0.0001$, corrected) (Figure 3 ). The involved nodal regions included two Default nodes (40 and 41), two Context nodes (2 and 7), three CinguloOperc nodes (7, 13, and 14), PERN_2, two DorsalAttn nodes (6 and 8$)$, four Visual nodes $(28,31,32$, and $34)$, SMHand_35, three SMMouth nodes $(6,7$, and 8$)$, and two Auditory nodes (1 and 2). There were no significant connections between FrontoParietal/Salience/VentralAttn/Subcortical nodes and other nodes.

3.2. Nodal Feature Analysis. Figures 4(a)-4(f) show the distribution of the hubs within 305 nodes for 81 subjects at the 


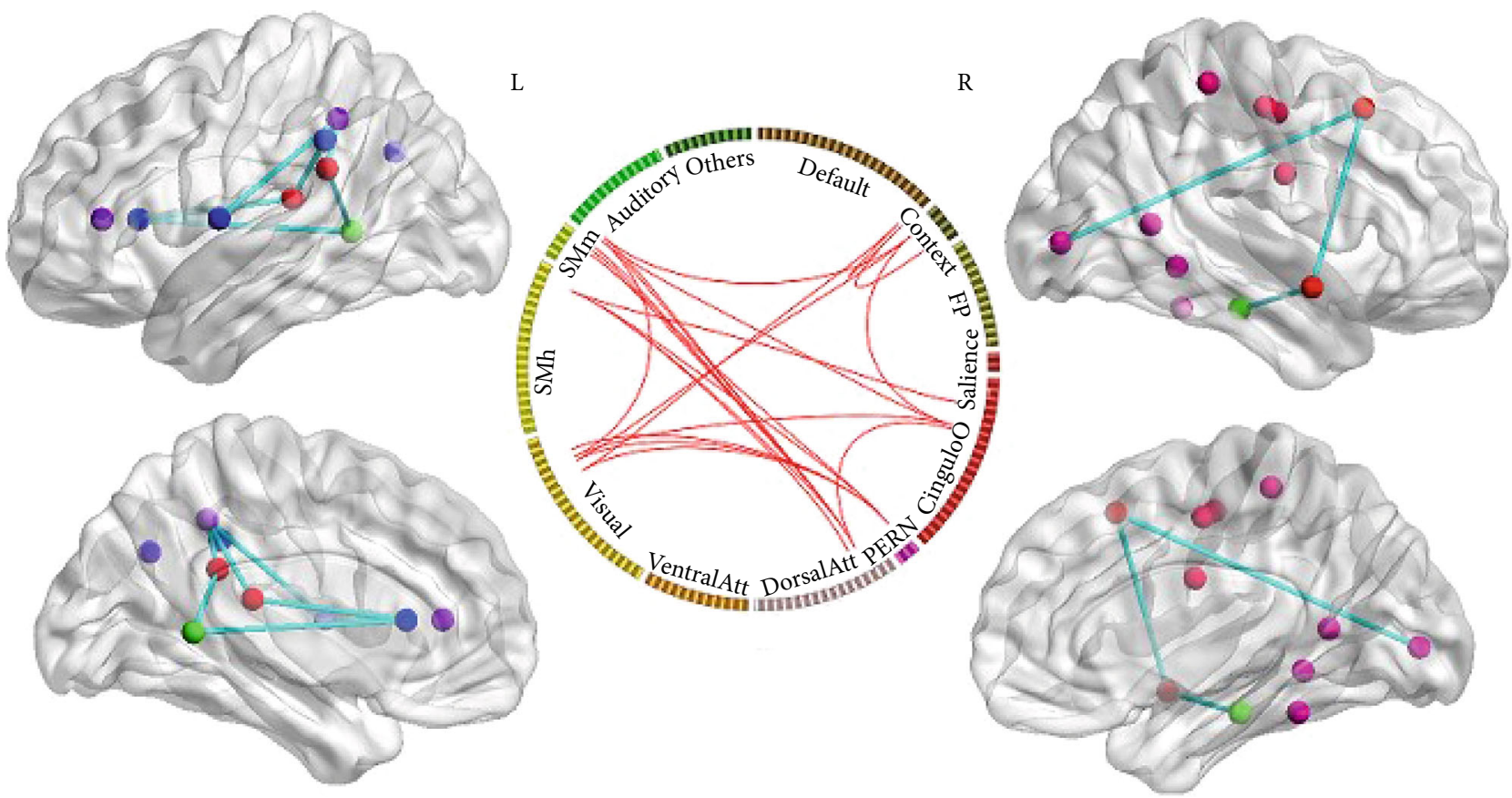

FIGURE 3: Two-dimension and three-dimension depictions of connectivity analysis result in the WM versus FIX condition. WM: working memory.

threshold of 10\%, and Figure 4(g) shows the mean ratio of the hubs across all 305 nodes at the 5-10\% threshold. Across each threshold, the number of the nonhubs was approximately $83 \%$ while the number of the hubs was approximately $17 \%$ in the FIX, EMOTION, and WM networks (Figure 4(g)). On the one hand, FIX networks only included provincial $(12.42 \% \pm 0.76 \%)$ and connector $(4.63 \% \pm 1.08 \%)$ hubs (Figures 4(a) and 4(b)) but no kinless hubs; EMOTION networks mainly included connector $(12.59 \% \pm 1.08 \%)$ and kinless hubs $(4.11 \% \pm 1.19 \%)$ (Figures $4(\mathrm{c})$ and $4(\mathrm{~d})$ ) but no provincial hubs; WM networks mainly covered kinless $(13.72 \% \pm 2.15 \%)$ and connector $(1.90 \% \pm 2.17 \%)$ hubs but no provincial hubs (Figures 4(e) and 4(f)). On the other hand, the ratio of the connector hubs increased with thresholds in FIX networks while the ratio of the kinless hubs increased with thresholds in EMOTION and WM networks. Moreover, ANOVA analyses indicated that the ratio of the connector hubs significantly increased in EMOTION networks relative to FIX and WM networks $(p<0.001)$ while the ratio of the kinless hubs significantly increased in WM networks compared to FIX and EMOTION networks $(p<0.001)$.

When we ignored the hubs whose subject ratio was less than 5\%, we found that the Context, Salience, and Subcortical communities did not include any hubs in the FIX condition. The most nodes of these communities shifted from nonhubs in the FIX condition to connector hubs in the EMOTION condition or to kinless hubs in the WM condition. However, Amygdala_1/2, Putamen_1, and Brain Stem became connector hubs only in the EMOTION condition. Additionally, when we considered the nodes as ROIs in Table 1, we found that Default_41, Context_2/7, FrontoParietal_3/20, Visual_ 28/31/32/34, SMmouth_6, and Auditory_1/2 in the FIX condition shifted from nonhubs of a single role to connector hubs in the EMOTION condition or to kinless hubs in the
WM condition (Figure 5). Similarly, Default_6/40, Ventra1Attn_11, DorsalAttn_8, and SMhand_35 in the FIX condition switched from provincial hubs of a single role to connector hubs in the EMOTION condition or to kinless hubs in the WM condition. PERN_2 and Visual_29 in the FIX and EMOTION condition changed their single role of connector hubs into kinless hubs in the WM condition. However, CinguloOperc_7/13/14/17/40, DorsalAttn_6, SMhand_1, and SMmouth_7/8 in the FIX condition turned their dual roles of provincial/connector hubs into connector hubs in the EMOTION condition or into kinless hubs in the WM condition.

3.3. Global Property Analysis. For FIX, EMOTION, and WM, ANOVA analyses on the global network properties showed significant task effects in global efficiency, transitivity, and assortativity $(p<0.0001)$ (Figure 6). Post hoc comparisons indicated that under the threshold of 5-15\%, FIX networks contained greater assortativity values than EMOTION networks while EMOTION networks showed greater assortativity values than WM networks $(p<0.001)$ (Figure 6(a)). Moreover, FIX networks exhibited greater global efficiency than EMOTION networks $(p<0.001)$ while EMOTION networks exhibited greater global efficiency than WM networks $(p<0.0001)$ (Figures 6(b) and 6(d)). Finally, FIX networks had greater transitivity values than EMOTION and WM networks $(p<0.0001)$ while WM networks included greater transitivity values than EMOTION networks $(p<0.001)$ (Figures 6(c) and 6(e)).

\section{Discussion}

In the present study, we used graph-theoretic approach to analyze the resting-state and task-state (WM and 


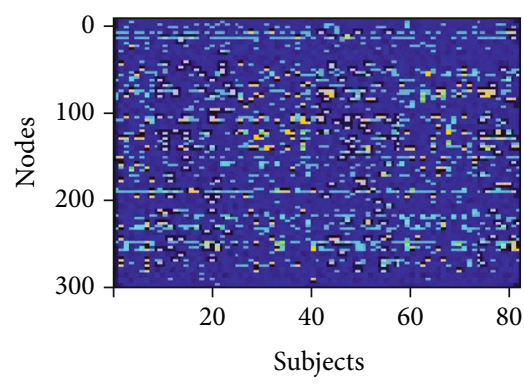

$\square$ Non-hub nodes $\square$ Provincial hubs $\square$ Connector hubs $\square$ Kinless hubs

(a)

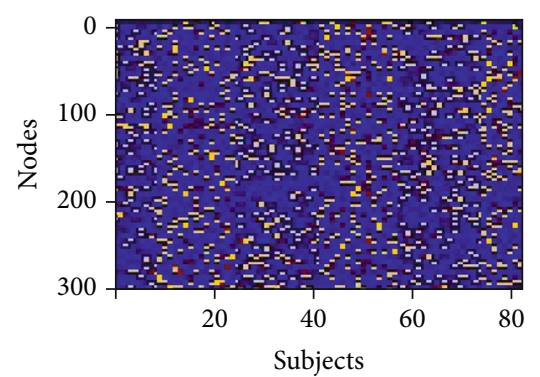

$\square$ Non-hub nodes $\square$ Provincial hubs $\square$ Connector hubs $\square$ Kinless hubs

(c)

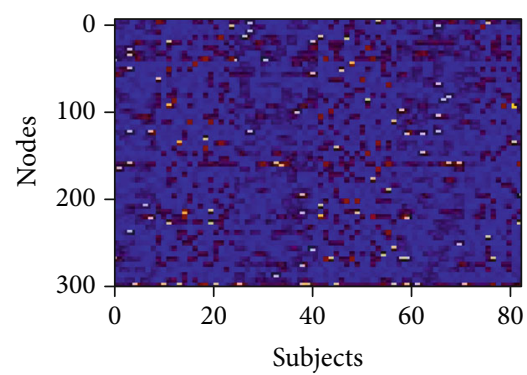

Non-hub nodes $\square$ Provincial hubs $\square$ Connector hubs $\square$ Kinless hubs

(e)

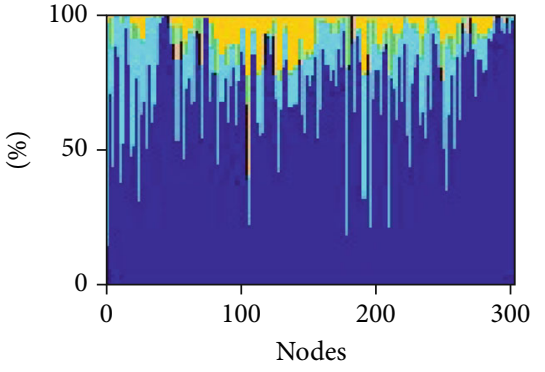

Non-hub nodes $\square$ Provincial hubs $\square$ Connector hubs $\square$ Kinless hubs

(b)

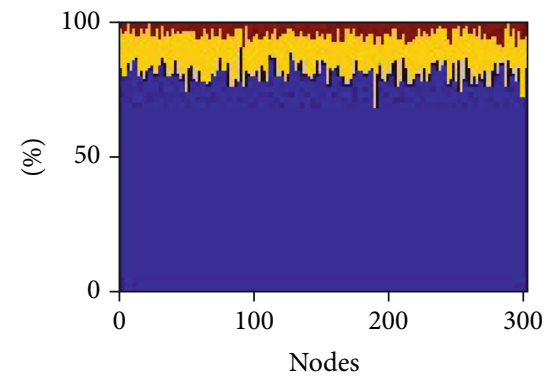

$\square$ Non-hub nodes $\square$ Provincial hubs

$\square$ Connector hubs $\square$ Kinless hubs

(d)

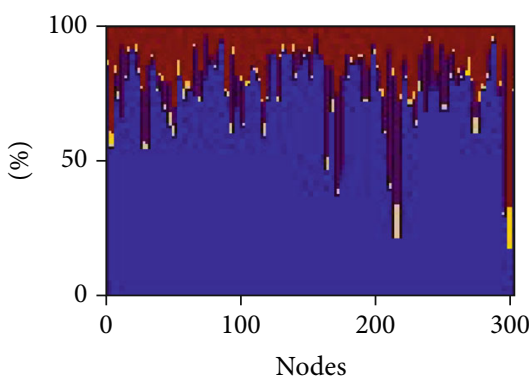

$\square$ Non-hub nodes $\square$ Provincial hubs

$\square$ Connector hubs $\square$ Kinless hubs

(f)

Figure 4: Continued. 


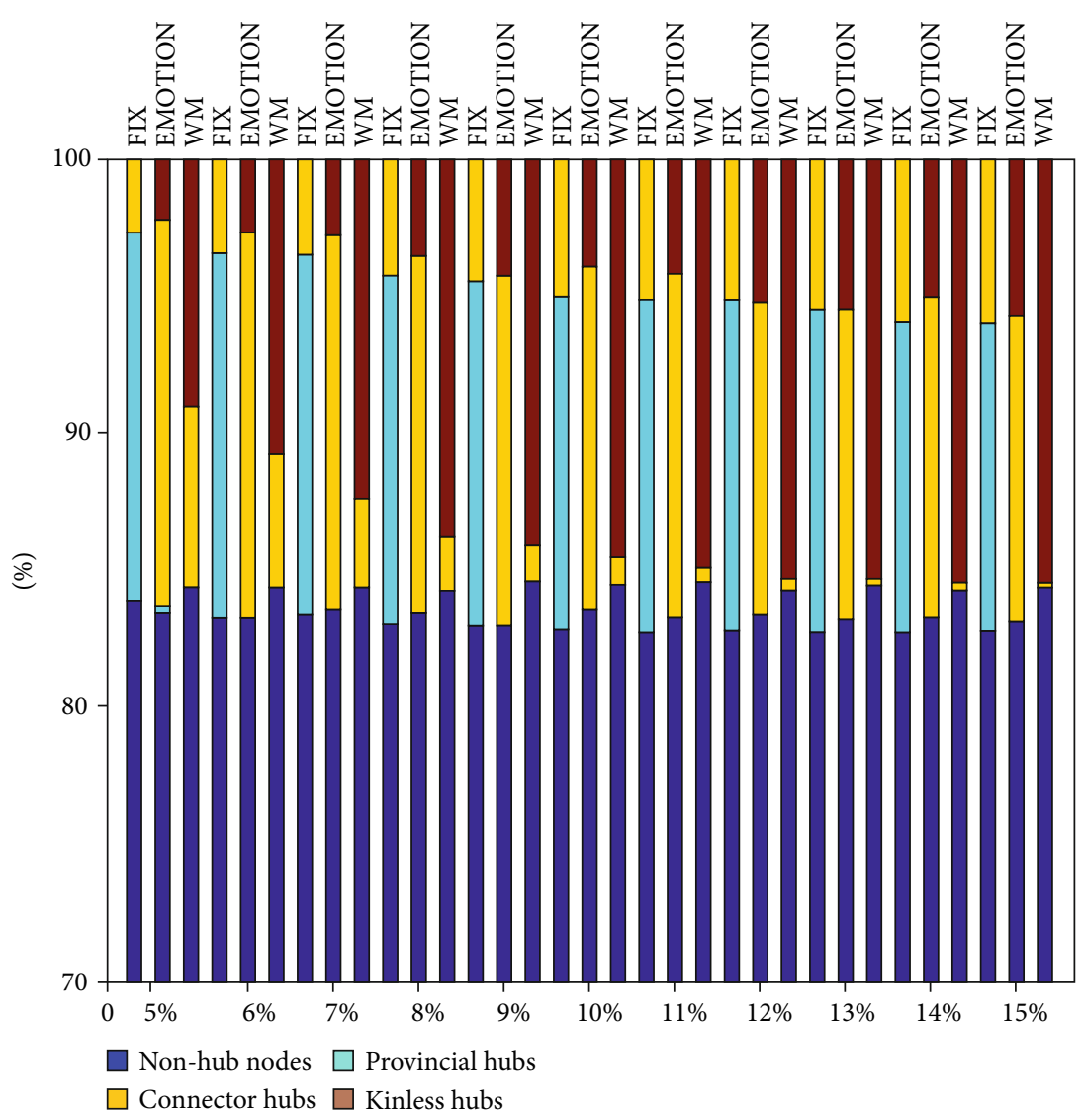

(g)

FIGURE 4: The hub distribution within 305 nodes for 81 subjects. (a, c, and e) Separately describe the hub distribution of FIX, EMOTION, and WM networks at the threshold of $10 \%$. (b, d, and f) Separately describe the subject ratio of each hub in FIX, EMOTION, and WM networks at the threshold of 10\%. (g) Describes the mean ratio of the hubs across all 305 nodes and all subjects at the 5-10\% threshold.

EMOTION) fMRI scans of 81 subjects from the HCP to determine how the topological properties of functional networks dynamically change according to task demands. Results indicated that relative to resting state, task demands significantly increase the strength of long-distance connections between modules but not within modules; the number of connector and kinless hubs significantly increases in EMOTION and WM networks while provincial hubs disappeared. Moreover, EMOTION and WM networks seem to become separated: their assortativity is close to zero and both the global efficiency and transitivity decreased. These results suggest that task demands change the architecture of intrinsic functional networks and cause local and global topological properties of functional networks at resting state to redistribute.

\subsection{Long-Distance Connections Dominate Intermodular} Communication at Task States. The NBS results indicated that the significant increase in connectivity strength occurred between different communities but not within modules at task state versus resting state. Long-distance connections occupy a relatively small ratio in functional networks [18]. However, a small quantity of long-distance connections is necessary to maintain intermodular information communi- cation because long-distance connection shorten the pathway of information transfer but does not significantly increase the wiring cost $[3,20,47]$. Consist with our results, previous MEEG studies also found that task demands (WM and motor performance) promote synchronization between brain networks through long-distance links [34, 40].

Moreover, WM and EMOTION networks show different connectivity patterns. Particularly, the long connection between Defualt_6 (Frontal_Sup_L) and VentralAttn_11 (Temporal_Mid_L) in EMOTION networks significantly increased while the long connections between Default_40/41 (Frontal_Mid_R and Temporal_Sup_R) and Visual_32/34 (Lingual_R and Fusiform_R) significantly increased in WM networks, which is consistent with the flexible reconfiguration in the interactions of DMN with other subnetworks [19]. However, there were no long connections between Subcortical nodes and other modular nodes. This may be partly attributed to very high threshold during the NBS analysis of fully linking networks. Taken together, long-distance connectivity patterns between modules have decisive significance for decoding multiple task-relevant information.

4.2. Connector and Kinless Hubs Dominate Task-State Functional Networks. Consistent with Finc et al. [13], we 


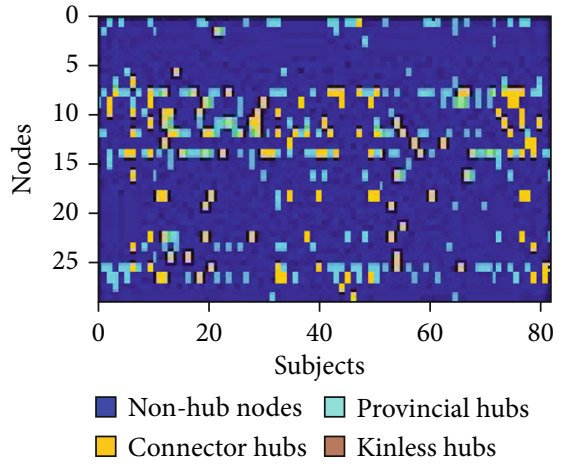

(a)

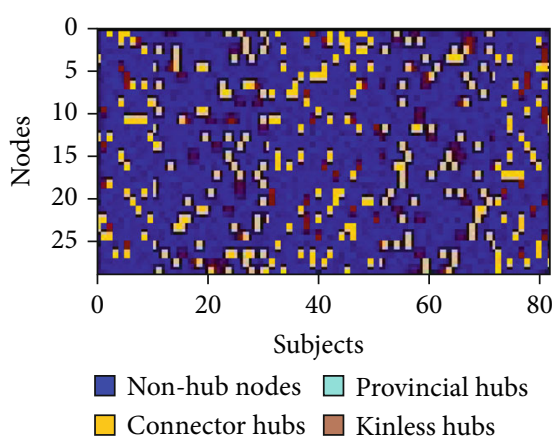

(c)

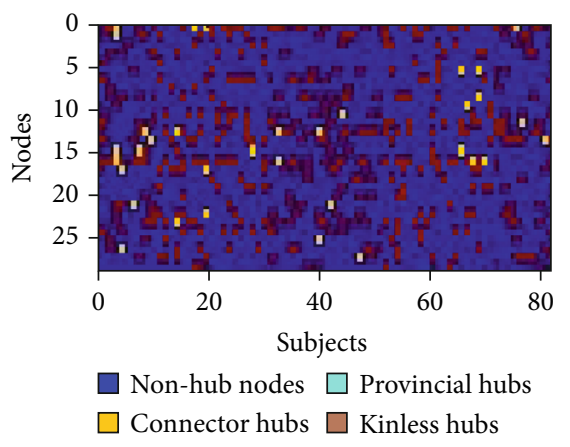

(e)

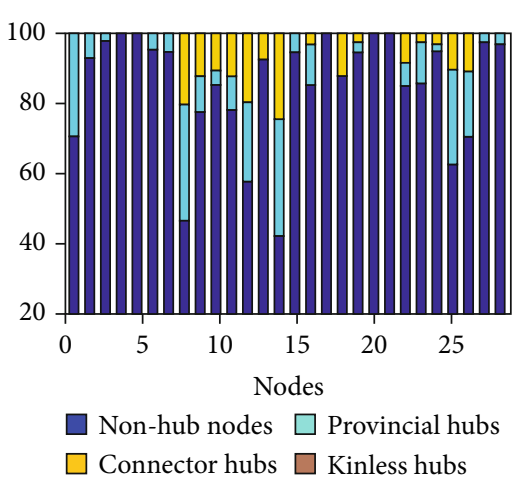

(b)

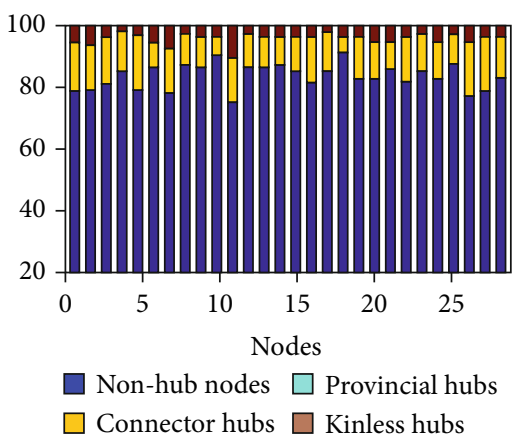

(d)

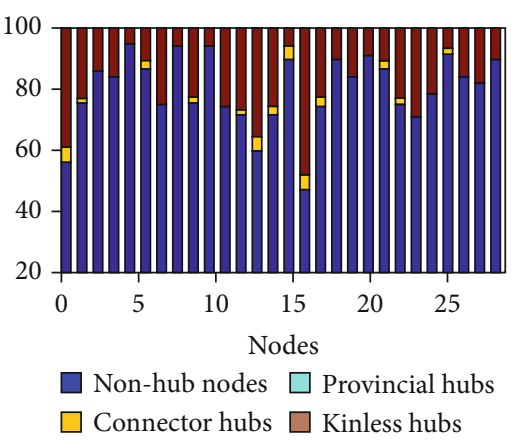

(f)

FIGURE 5: The hub distribution within 28 nodes of FIX (a), EMOTION (c), and WM (e) networks for 81 subjects and the subject ratio of each hub in FIX (b), EMOTION (d), and WM (f) at the threshold of $10 \%$. The nodal order is consistent with Table 1 . WM: working memory.

found that the number of connector hubs that have many links to most of the other modules increased in the EMOTION and WM networks relative to the FIX network. To be noted, kinless hubs that have links homogeneously distributing among all modules also increased, particularly in WM networks. However, kinless hubs did not appear in FIX networks, which might explain why kinless hubs were almost ignored in the literature related to functional networks. In addition, provincial hubs that have the vast majority of links within their own module mainly appeared in FIX networks but disappeared in EMOTION and WM networks. The previous MEG research also found that the motor tapping task causes the shift from resting-state networks dominated by provincial hubs to motor networks with a larger number of connector hubs [38]. Thus, consistent with the flexible hub theory [25], task demands need more between-module infor- mation communication so that connector and kinless hubs dominate task-state functional networks.

When we neglected the hubs whose subject ratios were less than 5\%, the hubs in EMOTION networks mainly consisted of connector hubs while the hubs in WM networks mainly belonged to kinless hubs. This might be attributed to the fact that PI values in WM networks were higher than those in EMOTION networks. This is also consistent with previous results indicating that more task demands need a more globally synchronized system to involve in [40]. Moreover, bilateral Amygdala, the left Putamen, and Brain Stem became connector hubs only in EMOTION networks, consistent with previous results [20, 26, 28-30], which implies that these hubs take a critical role in decoding emotional information. To be noted, in FIX networks, nonhubs (e.g., Defualt_41, Context_2/7, FrontoParietal_3/20, Visual_ 


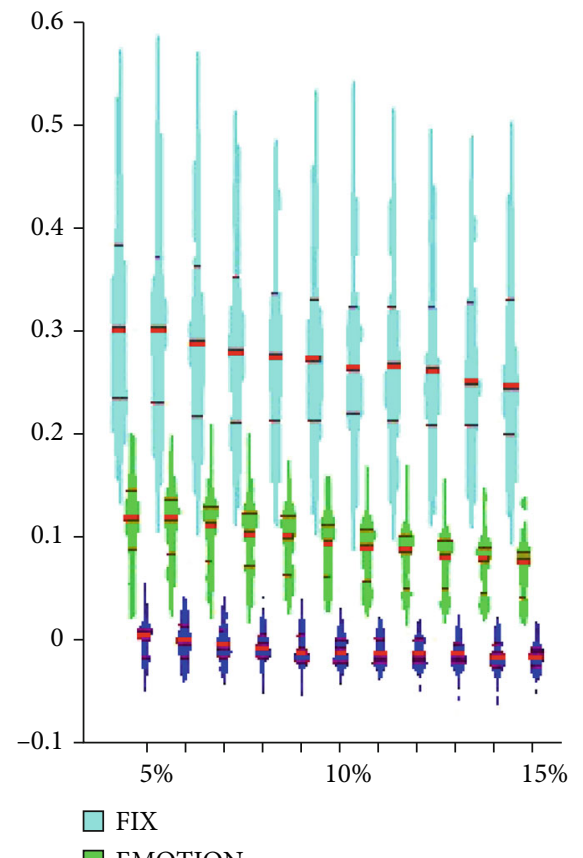

$\square$ EMOTION

$\square \mathrm{WM}$

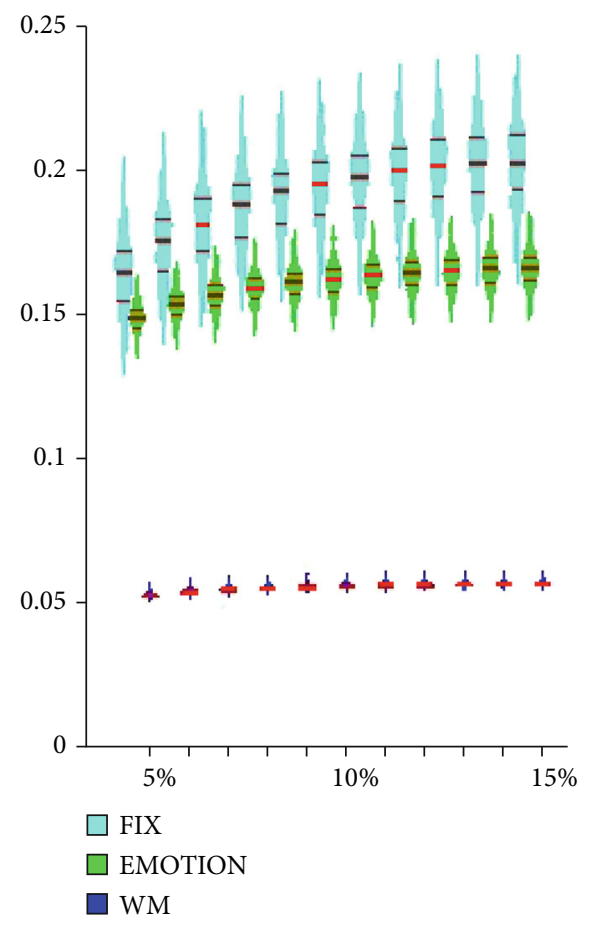

(a)

(b)


(c)

(d)

Figure 6: Continued. 


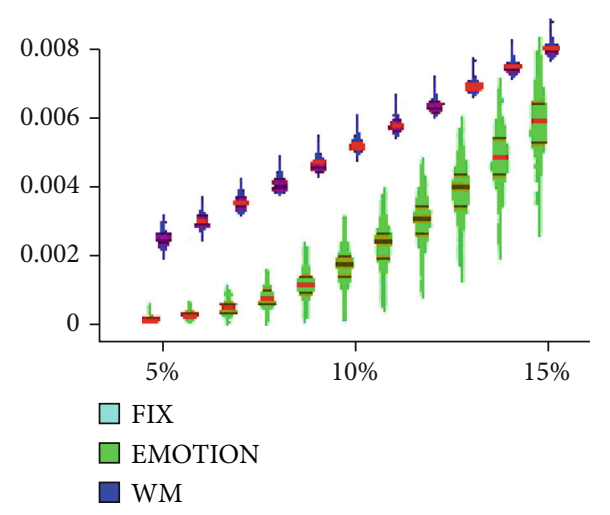

(e)

FIGURE 6: The ANOVA results of global property analysis for FIX, EMOTION, and WM: (a) assortativity; (b) global efficiency; (c) transitivity; (d) global efficiency graph enlarged for WM; (e) transitivity graph enlarged for EMOTION and WM at the threshold of 10\%. WM: working memory.

28/31/32/34, SMmouth_6, and Auditory_1/2) switch to connector hubs in EMOTION networks or kinless hubs in WM networks. These results suggest that task-relevant functional nodes dynamically reconfigure and shift their network roles from within-module to between-module.

\subsection{Functional Networks at Task State Tend to Be More} Separated Than Those at Resting State. Intrinsic functional networks at resting state represent a standard architecture and maintain the balance between integration and separation, which is evoked by task-relevant network changes $[6,14]$. Although less than 24 long-distance connections appeared and less than $17 \%$ functional nodes switched their roles in EMOTION and WM as discussed above, the global properties changed significantly. The global efficiency and transitivity significantly decreased in EMOTION and WM versus FIX, which means that task demands cause increase in pathway lengths (e.g., long connections appear) and decrease in clustering coefficients. This is inconsistent with increases in task demands leading to more integrated brain networks $[36,37]$. The previous study used a binary network to compute global properties [36] while our study used weighted networks. Undoubtedly, weighted correlation networks occupied more accurate representation than the binary networks.

What is more, the assortativity values reflecting a correlation coefficient between the degrees of all nodes significantly decreased and were close to zero at task state. Similarly, previous results also found that affective networks have negative assortativity and lower global efficiency and exhibit weaker small-worldness [30]. These results suggest that task demands break the balance between local and distant functional couplings at resting state [16] and cause functional networks to reconfigure their topologies. As a result, functional networks at task state tend to become more separated or random, a shift of network architecture to a more random configuration at higher levels of task difficulty $[8,30,40]$. Our result showing that more kinless hubs appeared in WM than in EMOTION networks to some degree provide direct evidence for this opinion. However, functional brain network topology was never completely randomized because of the constraint of structural network [8]. These explanations are not in agreement with previous MEG/EEG results that the clustering coefficient was conserved over a wide range of frequencies and increasing memory load increased clustering coefficient $[38,39]$. One possibility is because of the difference in signal measurement nature between fMRI and MEEG. Another possible interpretation is because the previous research absolutized the correlation between wavelet coefficients for each pair of sensors [38] or used EEG phase synchronization (positive and negative value) as a functional connectivity index [39], while the present study only contained positive connections. To clarify these inconsistencies, future studies necessarily combine fMRI with MEEG and select hard- and softthresholding approach of functional networks [48].

In summary, task demands break the balance between local and global coupling among brain regions in intrinsic functional networks. Long-distance functional connections dominated intermodular communication of functional networks at task states under high threshold. Correspondently, task-relevant connector or kinless hubs between modules were flexibly redistributed to promote task performance while provincial hubs within modules disappeared. Moreover, task-state networks significantly change their global topologies and tend to become more random. These observations provide important and direct evidences for the understanding of the organization principle behind the functional architecture of taskstate networks. To be cautious, it is unclear whether our results can be generalized to other task-state network. Future studies should use more other tasks with other network properties to further confirm our observations.

\section{Data Availability}

The data that support the findings of this study are available from the corresponding author upon reasonable request.

\section{Ethical Approval}

All procedures performed in the study were in accordance with the ethical standards of both the HCP and the research 
committee of Hengyang Normal University and with the 1964 Helsinki declaration and its later amendments or comparable ethical standards.

\section{Consent}

Informed consent was obtained from all individual participants included in the study.

\section{Conflicts of Interest}

All the authors declared no conflict of interest.

\section{Authors' Contributions}

All authors contributed to the study conception and design and performed the material preparation, data collection, and analysis. The first draft of the manuscript was written by Wenhai Zhang, and all authors commented on previous versions of the manuscript. All authors read and approved the final manuscript.

\section{Acknowledgments}

This study was funded by the National Natural Scientific Foundation of China (31470997 and 81171289), Jiangsu Provincial Social Science Foundation of China (19JYD009), and Hunan Provincial Natural Science Foundation of China (2018JJ2008).

\section{References}

[1] V. Menon, "Large-scale brain networks and psychopathology: a unifying triple network model," Trends in Cognitive Sciences, vol. 15, no. 10, pp. 483-506, 2011.

[2] D. van Essen, K. Ugurbil, E. Auerbach et al., "The human connectome project: a data acquisition perspective," NeuroImage, vol. 62, no. 4, pp. 2222-2231, 2012.

[3] R. L. Buckner, J. Sepulcre, T. Talukdar et al., "Cortical hubs revealed by intrinsic functional connectivity: mapping, assessment of stability, and relation to alzheimer's disease," Journal of Neuroscience, vol. 29, no. 6, pp. 1860-1873, 2009.

[4] D. S. Bassett and O. Sporns, "Network neuroscience," Nature Neuroscience, vol. 20, no. 3, pp. 353-364, 2017.

[5] C. Gratton, E. M. Nomura, F. Pérez, and M. D'Esposito, "Focal brain lesions to critical locations cause widespread disruption of the modular organization of the brain," Journal of Cognitive Neuroscience, vol. 24, no. 6, pp. 1275-1285, 2012.

[6] M. . W. Cole, D. . S. Bassett, J. . D. Power, T. . S. Braver, and S. . E. Petersen, "Intrinsic and task-evoked network architectures of the human brain," Neuron, vol. 83, no. 1, pp. 238$251,2014$.

[7] A. Fornito, A. Zalesky, and M. Breakspear, "Graph analysis of the human connectome: promise, progress, and pitfalls," NeuroImage, vol. 80, pp. 426-444, 2013.

[8] M. Rubinov and O. Sporns, "Complex network measures of brain connectivity: uses and interpretations," NeuroImage, vol. 52, no. 3, pp. 1059-1069, 2010.

[9] M. Girvan and M. E. J. Newman, "Community structure in social and biological networks," Proceedings of the National Academy of Sciences, vol. 99, no. 12, pp. 7821-7826, 2002.
[10] X. Liang, Q. Zou, Y. He, and Y. Yang, "Coupling of functional connectivity and regional cerebral blood flow reveals a physiological basis for network hubs of the human brain," PNAS, vol. 110, no. 5, pp. 1929-1934, 2013.

[11] X. N. Zuo, R. Ehmke, M. Mennes et al., "Network centrality in the human functional connectome," Cerebral Cortex, vol. 22, no. 8, pp. 1862-1875, 2012.

[12] R. F. Betzel, J. D. Medaglia, L. Papadopoulos et al., “The modular organization of human anatomical brain networks: accounting for the cost of wiring," Network Neuroscience, vol. 1, no. 1, pp. 42-68, 2017.

[13] K. Finc, K. Bonna, M. Lewandowska et al., "Transition of the functional brain network related to increasing cognitive demands," Human Brain Mapping, vol. 38, no. 7, pp. 36593674, 2017.

[14] C. Gratton, T. O. Laumann, E. M. Gordon, B. Adeyemo, and S. E. Petersen, "Evidence for two independent factors that modify brain networks to meet task goals," Cell Reports, vol. 17 , no. 5, pp. 1276-1288, 2016.

[15] A. M. Hermundstad, D. S. Bassett, K. S. Brown et al., "Structural foundations of resting-state and task-based functional connectivity in the human brain," Proceedings of the National Academy of Sciences, vol. 110, no. 15, pp. 61696174, 2013.

[16] J. Sepulcre, H. Liu, T. Talukdar, I. Martincorena, B. T. T. Yeo, and R. L. Buckner, "The organization of local and distant functional connectivity in the human brain," PLoS Computational Biology, vol. 6, no. 6, article e1000808, 2010.

[17] X. di, S. Gohel, E. H. Kim, and B. B. Biswal, "Task vs. rest-different network configurations between the coactivation and the resting-state brain networks," Frontiers in Human Neuroscience, vol. 7, no. 2, 2013.

[18] N. A. Crossley, A. Mechelli, P. E. Vertes et al., "Cognitive relevance of the community structure of the human brain functional coactivation network," Proceedings of the National Academy of Sciences, vol. 110, no. 28, pp. 1158311588, 2013.

[19] D. Vatansever, D. K. Menon, A. E. Manktelow, B. J. Sahakian, and E. A. Stamatakis, "Default mode dynamics for global functional integration," Journal of Neuroscience, vol. 35, no. 46, pp. 15254-15262, 2015.

[20] C. Sripada, M. Angstadt, D. Kessler et al., "Volitional regulation of emotions produces distributed alterations in connectivity between visual, attention control, and default networks," NeuroImage, vol. 89, no. 5, pp. 110-121, 2014.

[21] N. T. Markov, M. Ercsey-Ravasz, C. Lamy et al., "The role of long-range connections on the specificity of the macaque interareal cortical network," Proceedings of the National Academy of Sciences, vol. 110, no. 13, pp. 5187-5192, 2013.

[22] M. Kaiser and C. C. Hilgetag, "Nonoptimal component placement, but short processing paths, due to long-distance projections in neural systems," PLoS Computional Biology, vol. 2, no. 7, article e95, 2006.

[23] A. Zalesky, L. Cocchi, A. Fornito, M. M. Murray, and E. Bullmore, "Connectivity differences in brain networks," NeuroImage, vol. 60, no. 2, pp. 1055-1062, 2012.

[24] M. P. V. D. van den Heuvel and A. O. Sporns, "Network hubs in the human brain," Trends in Cognitive Sciences, vol. 17, no. 12, pp. 683-696, 2013.

[25] M. W. Cole, J. R. Reynolds, J. D. Power, G. Repovs, A. Anticevic, and T. S. Braver, "Multi-task connectivity reveals 
flexible hubs for adaptive task control," Nature Neuroscience, vol. 16, no. 9, pp. 1348-1355, 2013.

[26] T. Osada, Y. Adachi, K. Miyamoto, K. Jimura, R. Setsuie, and Y. Miyashita, "Dynamically allocated hub in task-evoked network predicts the vulnerable prefrontal locus for contextual memory retrieval in macaques," PLoS Biology, vol. 13, no. 6, article e1002177, 2015.

[27] R. Guimerà and L. A. Nunes Amaral, "Functional cartography of complex metabolic networks," Nature, vol. 433, no. 7028, pp. 895-900, 2005.

[28] U. Braun, A. Schäfer, H. Walter et al., "Dynamic reconfiguration of frontal brain networks during executive cognition in humans," Proceedings of the National Academy of Sciences, vol. 112, no. 37, pp. 11678-11683, 2015.

[29] K. A. Lindquist and L. F. Barrett, "A functional architecture of the human brain: emerging insights from the science of emotion," Trends in Cognitive Sciences, vol. 16, no. 11, pp. 533540, 2012.

[30] W. Zhang, H. Li, and X. Pan, "Positive and negative affective processing exhibit dissociable functional hubs during the viewing of affective pictures," Human Brain Mapping, vol. 36, no. 2, pp. 415-426, 2015.

[31] O. Sporns, "The human connectome: a complex network," Annals of the New York Academy of Sciences, vol. 1224, no. 1, pp. 109-125, 2011.

[32] B. M. Tijms, P. Seriès, D. J. Willshaw, and S. M. Lawrie, "Similarity-based extraction of individual networks from gray matter mri scans," Cerebral Cortex, vol. 22, no. 7, pp. 1530-1541, 2012.

[33] C. Yan, G. Gong, J. Wang et al., "Sex- and brain size-related small-world structural cortical networks in young adults: a DTI tractography study," Cerebral Cortex, vol. 21, no. 2, pp. 449-458, 2011.

[34] D. S. Bassett, E. T. Bullmore, A. Meyer-Lindenberg, J. A. Apud, D. R. Weinberger, and R. Coppola, "Cognitive fitness of costefficient brain functional networks," Proceedings of the National Academy of Sciences, vol. 106, no. 28, pp. 1174711752, 2009.

[35] O. Sporns and C. J. Honey, "Small worlds inside big brains," Proceedings of the National Academy of Sciences, vol. 103, no. 51, pp. 19219-19220, 2006.

[36] M. Alavash, P. Doebler, H. Holling, C. M. Thiel, and C. Gießing, "Is functional integration of resting state brain networks an unspecific biomarker for working memory performance?," NeuroImage, vol. 108, pp. 182-193, 2015.

[37] A. Fornito, J. Yoon, A. Zalesky, E. T. Bullmore, and C. S. Carter, "General and specific functional connectivity disturbances in first-episode schizophrenia during cognitive control performance," Biological Psychiatry, vol. 70, no. 1, pp. 64-72, 2011.

[38] D. S. Bassett, A. Meyer-Lindenberg, S. Achard, T. Duke, and E. Bullmore, "Adaptive reconfiguration of fractal small-world human brain functional networks," Proceedings of the National Academy of Sciences, vol. 103, no. 51, pp. 19518-19523, 2006.

[39] L. Li, J. X. Zhang, and T. Jiang, "Visual working memory loadrelated changes in neural activity and functional connectivity," PLoS One, vol. 6, no. 7, article e22357, 2011.

[40] M. G. Kitzbichler, R. N. A. Henson, M. L. Smith, P. J. Nathan, and E. T. Bullmore, "Cognitive effort drives workspace configuration of human brain functional networks," Journal of Neuroscience, vol. 31, no. 22, pp. 8259-8270, 2011.
[41] M. F. Glasser, S. N. Sotiropoulos, J. A. Wilson et al., "The minimal preprocessing pipelines for the Human Connectome Project," NeuroImage, vol. 80, pp. 105-124, 2013.

[42] A. R. Hariri, A. Tessitore, V. S. Mattay, F. Fera, and D. R. Weinberger, "The amygdala response to emotional stimuli: a comparison of faces and scenes," NeuroImage, vol. 17, no. 1, pp. 317-323, 2002.

[43] D. M. Barch, G. C. Burgess, M. P. Harms et al., "Function in the human connectome: task-fMRI and individual differences in behavior," NeuroImage, vol. 80, pp. 169-189, 2013.

[44] H. Cao, M. M. Plichta, A. Schäfer et al., "Test-retest reliability of fMRI-based graph theoretical properties during working memory, emotion processing, and resting state," NeuroImage, vol. 84, pp. 888-900, 2014.

[45] E. M. Gordon, T. O. Laumann, B. Adeyemo, J. F. Huckins, W. M. Kelley, and S. E. Petersen, "Generation and evaluation of a cortical area parcellation from resting-state correlations," Cerebral Cortex, vol. 26, no. 1, pp. 288-303, 2015.

[46] M. Rubinov and O. Sporns, "Weight-conserving characterization of complex functional brain networks," NeuroImage, vol. 56, no. 4, pp. 2068-2079, 2011.

[47] L. F. Barrett and A. B. Satpute, "Large-scale brain networks in affective and social neuroscience: towards an integrative functional architecture of the brain," Current Opinion in Neurobiology, vol. 23, no. 3, pp. 361-372, 2013.

[48] A. J. Schwarz and J. Mcgonigle, "Negative edges and soft thresholding in complex network analysis of resting state functional connectivity data," NeuroImage, vol. 55, no. 3, pp. 1132 1146, 2011. 